\title{
Inequalities and sufficient conditions for exponen- tial stability and instability for nonlinear Volterra difference equations with variable delay
}

\author{
ERNEST YANKSON (iD \\ Department of Mathematics, University \\ of Cape Coast, Ghana. \\ ernestoyank@gmail.com
}

\begin{abstract}
Inequalities and sufficient conditions that lead to exponential stability of the zero solution of the variable delay nonlinear Volterra difference equation

$$
x(n+1)=a(n) h(x(n))+\sum_{s=n-g(n)}^{n-1} b(n, s) h(x(s))
$$

are obtained. Lyapunov functionals are constructed and employed in obtaining the main results. A criterion for the instability of the zero solution is also provided. The results generalizes some results in the literature.
\end{abstract}

\section{RESUMEN}

Se obtienen desigualdades y condiciones suficientes que implican la estabilidad exponencial de la solución cero de la ecuación en diferencias no lineal de Volterra con retardo variable

$$
x(n+1)=a(n) h(x(n))+\sum_{s=n-g(n)}^{n-1} b(n, s) h(x(s)) .
$$

Se construyen funcionales de Lyapunov y se utilizan para obtener los resultados principales. Se entrega también un criterio para la inestabilidad de la solución cero. Los resultados generalizan algunos resultados en la literatura.

Keywords and Phrases: Exponential stability, Lyapunov functional, Instability.

2020 AMS Mathematics Subject Classification: 34D20, 34D40, 34K20. 


\section{Introduction}

Let $\mathbb{R}$ and $\mathbb{Z}^{+}$denote the set of real numbers and the set of positive integers respectively. In recent times, research into the stability properties of solutions of difference equations have gained the attention of many Mathematicians, see [1], [2], [4], [6], [7], [8] and the references cited therein. We are mainly motivated by the work of Kublik and Raffoul in [6] in which the authors obtained inequalities that lead to the exponential stability of the zero solution of the linear Volterra difference equation with finite delay

$$
x(n+1)=a(n) x(n)+\sum_{s=n-r}^{n-1} b(n, s) x(s),
$$

for some positive constant $r$.

In this paper we consider the scalar nonlinear Volterra difference equation with variable delay

$$
x(n+1)=a(n) h(x(n))+\sum_{s=n-g(n)}^{n-1} b(n, s) h(x(s)),
$$

where $a: \mathbb{Z}^{+} \rightarrow \mathbb{R}, b: \mathbb{Z}^{+} \times\left[-g_{0}, \infty\right) \rightarrow \mathbb{R}, h: \mathbb{R} \rightarrow \mathbb{R}$ and $0<g(n) \leq g_{0}$, for all $n \in \mathbb{Z}^{+}$for some positive constant $g_{0}$. We will obtain some inequalities regarding the solutions of (1.2) by employing Lyapunov functionals. These inequalities can be used to deduce exponential stability of the zero solution. Also, by means of a Lyapunov functional an instability criterion of the zero solution of equation (1.2) will be provided.

Let $\psi:\left[-g_{0}, 0\right] \rightarrow(-\infty, \infty)$ be a given bounded initial function with

$$
\|\psi\|=\max _{-g_{0} \leq s \leq 0}|\psi(s)| .
$$

We further denote the norm of a function $\varphi:\left[-g_{0}, \infty\right) \rightarrow(-\infty, \infty)$ by

$$
\|\varphi\|=\sup _{-g_{0} \leq s \leq \infty}|\varphi(s)| .
$$

Throughout this paper we let

$$
h(x)=x h_{1}(x)
$$

The notation $x_{n}$ means that $x_{n}(\tau)=x(n+\tau), \tau \in\left[-g_{0}, 0\right]$ as long as $x(n+\tau)$ is defined. Thus, $x_{n}$ is a function mapping an interval $\left[-g_{0}, 0\right]$ into $\mathbb{R}$. We say that $x(n) \equiv x\left(n, n_{0}, \psi\right)$ is a solution of (1.2) if $x(n)$ satisfies (1.2) for $n \geq n_{0}$ and $x_{n_{0}}=x\left(n_{0}+s\right)=\psi(s), s \in\left[-g_{0}, 0\right]$.

In this paper we use the convention that $\sum_{s=a}^{b} h(s)=0$ if $a>b$. The following notation is introduced.

Let

$$
A(n, s)=\sum_{u=n-s}^{\gamma} b(u+s, s), \text { where } 0<\gamma \leq g(n-1) \text { for all } n \in \mathbb{Z}^{+}
$$


It follows from (1.3) that

$$
A(n, n-g(n-1)-1)=0 .
$$

We assume throughout the paper that

$$
\Delta_{n} A^{2}(n, z) \leq 0, \text { for all } n+s+1 \leq z \leq n-1 .
$$

Due to (1.3) we can express (1.2) in the equivalent form

$$
\begin{aligned}
\Delta x(n)= & \left(a(n) h_{1}(x(n))+A(n+1, n) h_{1}(x(n))-1\right) x(n) \\
& -\Delta_{n} \sum_{s=n-g(n-1)-1}^{n-1} A(n, s) h(x(s)) .
\end{aligned}
$$

Definition 1.1. The zero solution of $(1.2)$ is said to be exponentially stable if any solution $x\left(n, n_{0}, \psi\right)$ of (1.2) satisfies

$$
\left|x\left(n, n_{0}, \psi\right)\right| \leq C\left(\|\psi\|, n_{0}\right) \zeta^{\gamma\left(n-n_{0}\right)}, \text { for all } n \geq n_{0},
$$

where $\zeta$ is a constant with $0<\zeta<1, C: \mathbb{R}^{+} \times \mathbb{Z}^{+} \rightarrow \mathbb{R}^{+}$, and $\gamma$ is a positive constant. The zero solution of (1.2) is said to be uniformly exponentially stable if $C$ is independent of $n_{0}$.

We end this section by stating a fact which will be used in the proof of Lemma 2.1, that is, if $u(n)$ is a sequence, then

$$
\Delta u^{2}(n)=u(n+1) \Delta u(n)+u(n) \Delta u(n)
$$

For more on the calculus of difference equations we refer to [3] and [5].

\section{Exponential Stability}

In this section we obtain inequalities that can be used to deduce the exponential stability of (1.2). To simplify notation we let

$$
Q(n, x)=(a(n)+A(n+1, n)) h_{1}(x(n))-1,
$$

and

$$
Q_{1}(n)=(a(n)+A(n+1, n))-1 .
$$

Lemma 2.1. Suppose that (1.3), (1.5) and for $\delta>0$,

$$
-\frac{\delta}{\delta g_{0}+g(n)} \leq Q(n, x) \leq-\delta g_{0} A^{2}(n+1, n) h_{1}^{2}(x(n))-Q^{2}(n, x),
$$


holds. If $1 \leq h_{1}(x)$, and

$$
V(n)=\left[x(n)+\sum_{s=n-g(n-1)-1}^{n-1} A(n, s) h(x(s))\right]^{2}+\delta \sum_{s=-g_{0}}^{-1} \sum_{z=n+s}^{n-1} A^{2}(n, z) h^{2}(x(z))
$$

then based on the solutions of (1.2) we have

$$
\Delta V(n) \leq Q_{1}(n) V(n) .
$$

Proof. Let $x\left(n, n_{0}, \psi\right)$ be a solution of (1.2) and let $V(n)$ be defined by (2.2). It must also be noted that in view of condition $(2.1), Q(n, x)<0$ for all $n \geq 0$. This together with the fact that $1 \leq h_{1}(x)$ also implies that $Q(n, x) \leq Q_{1}(n)<0$. Then based on the solutions of (1.2) we have

$$
\begin{aligned}
\Delta V(n)= & {\left[x(n+1)+\sum_{s=n-g(n)}^{n} A(n+1, s) h(x(s))\right] } \\
& \times \Delta\left[x(n)+\sum_{s=n-g(n-1)-1}^{n-1} A(n, s) h(x(s))\right] \\
& +\left[x(n)+\sum_{s=n-g(n-1)-1}^{n-1} A(n, s) h(x(s))\right] \\
& \times \Delta\left[x(n)+\sum_{s=n-g(n-1)-1}^{n-1} A(n, s) h(x(s))\right] \\
& +\delta \Delta_{n} \sum_{s=-g_{0}}^{-1} \sum_{z=n+s}^{n-1} A^{2}(n, z) h^{2}(x(z)) .
\end{aligned}
$$

But

$$
\begin{aligned}
& x(n+1)+\sum_{s=n-g(n)}^{n} A(n+1, s) h(x(s)) \\
= & (Q(n, x)+1) x(n)-\Delta_{n} \sum_{s=n-g(n-1)-1}^{n-1} A(n, s) h(x(s))+\sum_{s=n-g(n)}^{n} A(n+1, s) h(x(s)) \\
= & (Q(n, x)+1) x(n)+\sum_{s=n-g(n-1)-1}^{n-1} A(n, s) h(x(s)) \\
= & (Q(n, x)+1) x(n)+\sum_{s=n-g(n)}^{n-1} A(n, s) h(x(s))
\end{aligned}
$$


where we have used the fact that $A(n, n-g(n-1)-1)=0$. Using $(2.5)$ in (2.4) we obtain

$$
\begin{aligned}
\Delta V(n)= & {\left.[Q(n, x)+1) x(n)+\sum_{s=n-g(n)}^{n-1} A(n, s) h(x(s))\right] Q(n, x) x(n) } \\
& +\left[x(n)+\sum_{s=n-g(n)}^{n-1} A(n, s) h(x(s))\right] Q(n, x) x(n) \\
& +\delta \Delta_{n} \sum_{s=-g_{0}} \sum_{z=n+s}^{n-1} A^{2}(n, z) h^{2}(x(z)) \\
= & Q(n, x) V(n)+\left(Q^{2}(n, x)+Q(n, x)\right) x^{2}(n)+\delta \Delta_{n} \sum_{s=-g_{0}}^{-1} \sum_{z=n+s}^{n-1} A^{2}(n, z) h^{2}(x(z)) \\
& -Q(n, x)\left(\sum_{s=n-g(n)}^{n-1} A(n, s) h(x(s))\right)^{2} \\
& -\delta Q(n, x) \sum_{s=-g_{0}}^{-1} \sum_{z=n+s}^{n-1} A^{2}(n, z) h^{2}(x(z))
\end{aligned}
$$

Considering the third term on the right hand side of (2.6) we obtain

$$
\begin{aligned}
& \Delta_{n} \sum_{s=-g_{0}}^{-1} \sum_{z=n+s}^{n-1} A^{2}(n, z) h^{2}(x(z)) \\
&= \sum_{s=-g_{0}}^{-1} \sum_{z=n+s+1}^{n} A^{2}(n+1, z) h^{2}(x(z))-\sum_{s=-g_{0}}^{-1} \sum_{z=n+s}^{n-1} A^{2}(n, z) h^{2}(x(z)) \\
&= \sum_{s=-g_{0}}^{-1}\left[A^{2}(n+1, n) h\left(x^{2}(n)\right)+\sum_{z=n+s+1}^{n-1} A^{2}(n+1, z) h^{2}(x(z))\right. \\
&\left.-\sum_{z=n+s+1}^{n-1} A^{2}(n, z) h^{2}(x(z))-A^{2}(n, n+s) h^{2}(x(n+s))\right] \\
&= \sum_{s=-g_{0}}^{-1}\left(A^{2}(n+1, n) h_{1}^{2}(x(n)) x^{2}(n)-A^{2}(n, n+s) h^{2}(x(n+s))\right) \\
&+\sum_{s=-g_{0}}^{-2} \sum_{z=n+s+1}^{n-1} \Delta_{n} A^{2}(n, z) h^{2}(x(z)) \\
&= g_{0} A^{2}(n+1, n) h_{1}^{2}(x(n)) x^{2}(n)-\sum_{s=-g_{0}}^{-1} A^{2}(n, n+s) h^{2}(x(n+s)) \\
&+\sum_{s=-g_{0}}^{-2} \sum_{z=n+s+1}^{n-1} \Delta_{n} A^{2}(n, z) h^{2}(x(z)) \\
& \leq g_{0} A^{2}(n+1, n) h_{1}^{2}(x(n)) x^{2}(n)-\sum_{s=-g_{0}}^{-1} A^{2}(n, n+s) h^{2}(x(n+s)) . \\
& g_{0}(n+1, n) h_{1}^{2}(x(n)) x^{2}(n)-\sum_{z=n-g_{0}}^{n-1} A^{2}(n, z) h^{2}(x(z))
\end{aligned}
$$


Applying the Holder's inequality to the squared term in the fourth term on the right hand side of (2.6) gives

$$
\begin{aligned}
\left(\sum_{s=n-g(n)}^{n-1} A(n, s) h(x(s))\right)^{2} & \leq g(n) \sum_{s=n-g(n)}^{n-1} A^{2}(n, s) h^{2}(x(s)) \\
& \leq g(n) \sum_{s=n-g_{0}}^{n-1} A^{2}(n, s) h^{2}(x(s)) .
\end{aligned}
$$

Considering the last term on the right hand side of (2.6) we obtain

$$
\sum_{s=-g_{0}}^{-1} \sum_{z=n+s}^{n-1} A^{2}(n, z) h^{2}(x(z)) \leq g_{0} \sum_{s=n-g_{0}}^{n-1} A^{2}(n, s) h^{2}(x(s))
$$

Substituting (2.7), (2.8) and (2.9) in (2.6) we obtain

$$
\begin{aligned}
\Delta V(n) \leq & Q(n, x) V(n)+\left(Q^{2}(n, x)+Q(n, x)+\delta g_{0} A^{2}(n+1, n) h_{1}^{2}(x(n))\right) x^{2}(n) \\
& +\left[-\left(g(n)+\delta g_{0}\right) Q(n, x)-\delta\right] \sum_{s=n-g_{0}}^{n-1} A^{2}(n, s) h^{2}(x(s)) \\
\leq & Q(n, x) V(n)+\left(Q^{2}(n, x)+Q(n, x)+\delta g_{0} A^{2}(n+1, n)\right) x^{2}(n) \\
& +\left[-\left(g(n)+\delta g_{0}\right) Q(n, x)-\delta\right] \sum_{s=n-g_{0}}^{n-1} A^{2}(n, s) h^{2}(x(s)) \\
\leq & Q(n, x) V(n) \\
\leq & Q_{1}(n) V(n) .
\end{aligned}
$$

Theorem 2.2. Suppose the hypothesis of Lemma 2.1 hold. Then any solution $x(n)=x\left(n, n_{0}, \psi\right)$ of (1.2) satisfies the exponential inequality

$$
|x(n)| \leq \sqrt{\frac{g_{0}+\delta}{\delta} V\left(n_{0}\right) \prod_{s=n_{0}}^{n-1}(a(n)+A(n+1, n))}
$$

for $n \geq n_{0}$.

Proof. Let $V(n)$ be defined by (2.2). Changing the order of summation in the second term on the right hand side of $(2.2)$ we obtain

$$
\begin{aligned}
\delta \sum_{s=-g_{0}}^{-1} \sum_{z=n+s}^{n-1} A^{2}(n, z) h^{2}(x(z)) & =\delta \sum_{z=n-g_{0}}^{n-1} \sum_{s=-g_{0}}^{z-n} A^{2}(n, z) h^{2}(x(z)) \\
& =\delta \sum_{z=n-g_{0}}^{n-1} A^{2}(n, z) h^{2}(x(z))\left(z-n+g_{0}+1\right) \\
& \geq \delta \sum_{z=n-g_{0}}^{n-1} A^{2}(n, z) h^{2}(x(z)) \\
& \geq \delta \sum_{z=n-g(n)}^{n-1} A^{2}(n, z) h^{2}(x(z))
\end{aligned}
$$


where we have used the fact that if $n-g_{0} \leq z \leq n-1$ then $1 \leq z-n+g_{0}+1 \leq g_{0}$ and $n-g_{0} \leq n-g(n)$.

Also, we note that

$$
\left(\sum_{z=n-g(n)}^{n-1} A(n, z) h(x(z))\right)^{2} \leq g_{0} \sum_{z=n-g(n)}^{n-1} A^{2}(n, z) h^{2}(x(z)) .
$$

Hence,

$$
\delta \sum_{s=-g_{0}}^{-1} \sum_{z=n+s}^{n-1} A^{2}(n, z) h^{2}(x(z)) \geq \frac{\delta}{g_{0}}\left(\sum_{z=n-g(n)}^{n-1} A(n, z) h(x(z))\right)^{2}
$$

Thus,

$$
\begin{aligned}
V(n) & \geq\left[x(n)+\sum_{s=n-g(n)}^{n-1} A^{2}(n, z) h^{2}(x(z))\right]^{2}+\frac{\delta}{g_{0}}\left(\sum_{z=n-g(n)}^{n-1} A(n, z) h(x(z))\right)^{2} \\
& =\frac{\delta}{g_{0}+\delta} x^{2}(n)+\left[\sqrt{\frac{g_{0}}{g_{0}+\delta}} x(n)+\sqrt{\frac{g_{0}+\delta}{g_{0}}} \sum_{z=n-g(n)}^{n-1} A(n, z) h(x(z))\right]^{2} \\
& \geq \frac{\delta}{g_{0}+\delta} x^{2}(n) .
\end{aligned}
$$

But

$$
V(n) \leq V\left(n_{0}\right) \prod_{s=n_{0}}^{n-1}((a(n)+A(n+1, n))
$$

This implies that

$$
\frac{\delta}{g_{0}+\delta} x^{2}(n) \leq V\left(n_{0}\right) \prod_{s=n_{0}}^{n-1}((a(n)+A(n+1, n))
$$

Hence,

$$
|x(n)| \leq \sqrt{\frac{g_{0}+\delta}{\delta} V\left(n_{0}\right) \prod_{s=n_{0}}^{n-1}(a(n)+A(n+1, n))} .
$$

This completes the proof.

Corollary 2.3. Suppose that the hypotheses of Theorem 3.2 hold. Suppose that there exists a positive number $\alpha<1$ such that

$$
0<a(n)+A(n+1, n) \leq \alpha
$$

Then the zero solution of (1.2) is exponentially stable. 
Proof. It follows from (2.10) that

$$
\begin{aligned}
|x(n)| & \leq \sqrt{\frac{g_{0}+\delta}{\delta} V\left(n_{0}\right) \prod_{s=n_{0}}^{n-1}(a(n)+A(n+1, n))} \\
& \leq \sqrt{\frac{g_{0}+\delta}{\delta} V\left(n_{0}\right) \alpha^{n-n_{0}}}
\end{aligned}
$$

for $n \geq n_{0}$. Since $\alpha \in(0,1)$ the proof is complete.

\section{$3 \quad$ Instability Criteria}

In this section we consider the problem of finding a criteria for instability of the zero solution of (1.2). A suitable Lyapunov functional will be used to obtain the instability criteria.

Theorem 3.1. Assume that (1.3), (1.5) hold and let $\rho>g_{0}$ be a constant. Assume that $Q_{1}(n)>0$ and $Q(n, x)>0$ such that

$$
Q^{2}(n, x)+Q(n, x)-\rho A^{2}(n+1, n) h_{1}^{2}(x(n)) \geq 0 .
$$

If $1 \leq h_{1}(x)$ and

$$
V(n)=\left[x(n)+\sum_{s=n-g(n-1)-1}^{n-1} A(n, s) h(x(s))\right]^{2}-\rho \sum_{s=n-g(n-1)-1}^{n-1} A^{2}(n, s) h^{2}(x(s))
$$

then, based on the solutions of (1.2) we have

$$
\Delta V(n) \geq Q_{1}(n) V(n) .
$$

Proof. Let $x\left(n, n_{0}, \psi\right)$ be a solution of $(1.2)$ and let $V(n)$ be defined by (3.2). Then based on the solutions of (1.2) we have

$$
\begin{aligned}
\Delta V(n)= & {\left[x(n+1)+\sum_{s=n-g(n)}^{n-1} A(n, s) h(x(s))\right] } \\
& \times \Delta\left[x(n)+\sum_{s=n-g(n-1)-1}^{n-1} A(n, s) h(x(s))\right] \\
& +\left[x(n)+\sum_{s=n-g(n-1)-1}^{n-1} A(n, s) h(x(s))\right] \\
& \times \Delta\left[x(n)+\sum_{s=n-g(n-1)-1}^{n-1} A(n, s) h(x(s))\right] \\
& -\rho\left[A^{2}(n+1, n) h^{2}(x(n))+\sum_{s=n-g(n)}^{n-1} \Delta_{n} A^{2}(n, s) h^{2}(x(s))\right]
\end{aligned}
$$




$$
\begin{aligned}
\geq & {\left.[Q(n, x)+1) x(n)+\sum_{s=n-g(n)}^{n-1} A(n, s) h(x(s))\right] Q(n, x) x(n) } \\
& +\left[x(n)+\sum_{s=n-g(n)}^{n-1} A(n, s) h(x(s))\right] Q(n, x) x(n) \\
& -\rho A^{2}(n+1, n) h^{2}(x(n)) \\
= & Q(n, x) V(n)+\left(Q^{2}(n, x)+Q(n, x)-\rho A^{2}(n+1, n) h_{1}^{2}(x(n))\right) x^{2}(n) \\
& -Q(n, x)\left(\sum_{s=n-g(n-1)-1}^{n-1} A(n, s) h(x(s))\right)^{2} \\
& +Q(n, x) \rho \sum_{s=n-g(n-1)-1}^{n-1} A^{2}(n, s) h^{2}(x(s)) \\
\geq & Q(n, x) V(n)+\left(Q^{2}(n, x)+Q(n, x)-\rho A^{2}(n+1, n) h_{1}^{2}(x(n))\right) x^{2}(n) \\
& +Q(n, x)\left(\rho-g_{0}\right) \sum_{s=n-g(n-1)-1}^{n-1} A^{2}(n, s) h^{2}(x(s)) \\
\geq & Q(n, x) V(n) \quad Q_{1}(n) V(n) .
\end{aligned}
$$

This completes the proof.

Theorem 3.2. Suppose the hypothesis of Theorem 3.1 hold. Then the zero solution of (1.2) is unstable, provided that

$$
\prod_{s=0}^{\infty}(a(n)+A(n+1, n))=\infty .
$$

Proof. We have from Theorem 3.1 that

$$
\Delta V(n) \geq Q_{1}(n) V(n),
$$

which implies that

$$
V(n) \geq V\left(n_{0}\right) \prod_{s=n_{0}}^{\infty}(a(s)+A(s+1, s)) .
$$

Using the definition of $V(n)$ in (3.2) we have that

$$
\begin{aligned}
V(n)= & x^{2}(n)+2 x(n) \sum_{s=n-g(n-1)-1}^{n-1} A(n, s) h(x(s)) \\
& +\left[\sum_{s=n-g(n-1)-1}^{n-1} A(n, s) h(x(s))\right]^{2}-\rho \sum_{s=n-g(n-1)-1}^{n-1} A^{2}(n, s) h^{2}(x(s))
\end{aligned}
$$

Now let $\beta=\rho-g_{0}$, then from

$$
\left(\frac{\sqrt{g_{0}}}{\sqrt{\beta}} a-\frac{\sqrt{\beta}}{\sqrt{g_{0}}} b\right)^{2} \geq 0
$$


we have

$$
2 a b \leq \frac{g_{0}}{\beta} a^{2}+\frac{\beta}{g_{0}} b^{2} .
$$

It follows from this inequality that

$$
\begin{aligned}
2 x(n) \sum_{s=n-g(n-1)-1}^{n-1} A(n, s) h(x(s)) & \leq 2|x(n)|\left|\sum_{s=n-g(n-1)-1}^{n-1} A(n, s) h(x(s))\right| \\
& \leq \frac{g_{0}}{\beta} x^{2}(n)+\frac{\beta}{g_{0}}\left[\sum_{s=n-g(n-1)-1}^{n-1} A(n, s) h(x(s))\right]^{2} \\
& \leq \frac{g_{0}}{\beta} x^{2}(n)+\frac{\beta}{g_{0}} g_{0} \sum_{s=n-g(n-1)-1}^{n-1} A^{2}(n, s) h^{2}(x(s)) .
\end{aligned}
$$

Substituting (3.5) into (3.4) we obtain

$$
\begin{aligned}
V(n) & \leq x^{2}(n)+\frac{g_{0}}{\beta} x^{2}(n)+\left(\beta+g_{0}-\rho\right) \sum_{s=n-g(n-1)-1}^{n-1} A^{2}(n, s) h^{2}(x(s)) \\
& =\frac{\beta+g_{0}}{\beta} x^{2}(n) \\
& \leq \frac{\rho}{\rho-g_{0}} x^{2}(n) .
\end{aligned}
$$

Using the last inequality and (3.3) we obtain

$$
\begin{aligned}
|x(n)|^{2} & \geq \frac{\rho-g_{0}}{\rho} V(n) \\
& =\frac{\rho-g_{0}}{\rho} V\left(n_{0}\right) \prod_{s=n_{0}}^{\infty}[a(n)+A(n+1, n)] .
\end{aligned}
$$

This completes the proof. 


\section{References}

[1] I. Berezansky, and E. Braverman, "Exponential stability of difference equations with several delays: Recursive approach", Adv. Difference Edu., article ID 104310, pp. 13, 2009.

[2] El-Morshedy, "New explicit global asymptotic stability criteria for higher order difference equations", vol. 336, no. 1, pp. 262-276, 2007.

[3] S. Elaydi, An introduction to Difference Equations, Springer Verlage, New York, 3rd Edition, 2005.

[4] M. Islam, and E. Yankson, "Boundedness and stability in nonlinear delay difference equations employing fixed point theory", Electron. J. Qual. Theory Differ. Equ., vol. 26, 2005.

[5] W. Kelley, and A. Peterson, Difference Equations: An Introduction with Applications, Second Edition, Academic Press, New York, 2001.

[6] C. Kublik, and Y. Raffoul, "Lyapunov functionals that lead to exponential stability and instability in finite delay Volterra difference equations", Acta Mathematica Vietnamica, vol. 41, pp. 77-89, 2016.

[7] Y. Raffoul, "Stability and periodicity in discrete delay equations", J. Math. Anal. Appl., vol. 324, pp. 1356-1362, 2006.

[8] Y. Raffoul, "Inequalities that lead to exponential stability and instability in delay difference equations", J. Inequal. Pure Appl. Math, vol. 10, no. 3, article 70, pp. 9, 2009. 